\title{
O PROCESSO DE TRABALHO EM SAÚDE DA FAMÍLIA NO CONTEXTO DO INTERIOR DAAMAZÔNIA
}

Iana Bárbara Ramalho Faria ${ }^{1}$, Júlia Carvalho Ventura ${ }^{1}$, Daniela Eda Silva² ${ }^{2}$ Rodrigo Otávio Moretti-Pires ${ }^{3}$

\begin{abstract}
RESUMO: Pesquisa exploratória com abordagem qualitativa objetivou apreender a percepção dos profissionais sobre o funcionamento da Estratégia Saúde da Família (ESF) no interior da Amazônia. Para a leitura dos dados, utilizou-se a hermenêutica dialética. Foram identificadas contradições entre o preconizado para a ESF e a operacionalização deste modelo de atenção: atenção às necessidades de saúde versus atendimento à demanda; vínculo profissional versus rotatividade da equipe; trabalho em equipe versus trabalho disciplinar; ausência de corresponsabilização entre Gestão Municipal e equipes da ESF. Conclui-se que há necessidade de políticas específicas para reorganização do funcionamento das gestões locais e municipais, com foco na ampla participação no processo de prestação de serviços.
\end{abstract}

PALAVRAS-CHAVE: Atenção primária à saúde; Prática profissional; Saúde da família.

\section{THE FAMILY HEALTH WORK PROCESS ON THE CONTEXT OF THE INTERIOR OF AMAZON}

\begin{abstract}
It's a exploratory research with a qualitative approach which aimed to apprehend the perception of the health care professionals about the development of the Family Health Strategy (FHS) on the interior of Amazon. For the results reading, the dialectic hermeneutic was used. Contradictions among what's recommended to the ESF and the operationalization of this model of care were identified: attention for the health needs versus attending the population demands; professional link versus health team turnover; team work versus discipline work; lack of sharing responsibilities between the municipality management team and the ESF teams. It's concluded that there's a need to build specific policies that enables the reorganization of the management team's work, both local and municipal, with focus in the wide participation on the health care services delivery.
\end{abstract}

KEYWORDS: Primary health care; Professional practice; Family health.

\section{EL PROCESO DE TRABAJO EN SALUD DE LA FAMILIA EN EL CONTEXTO DEL INTERIOR DE LAAMAZONIA}

RESUMEN: Investigación exploratoria con abordaje cualitativa objetivó aprehender la percepción de los profesionales sobre el funcionamiento de la Estrategia Salud de la Familia (ESF) en el interior de la Amazonia. Para la lectura de los datos utilizó la hermenéutica dialéctica. Fueron identificadas contradicciones entre lo preconizado para la ESF y la operacionalización de este modelo de atención: atención a las necesidades de salud versus atendimiento a la demanda; vínculo profesional versus rotación del equipo; trabajo en equipo versus trabajo disciplinar; ausencia de coresponsabilización entre Gestión Municipal y equipos de la ESF. Conclúyese que hay necesidad de políticas específicas para reorganización del funcionamiento de las gestiones, locales y municipales, con enfoque en la amplia participación en el proceso de prestación de servicios.

PALABRAS CLAVE: Atención primaria a la salud; Práctica profesional; Salud de la familia.

${ }^{1}$ Acadêmica do Curso de Graduação em Nutrição da Universidade Federal de Santa Catarina-UFSC.

${ }^{2}$ Enfermeira. Mestranda do Programa de Pós-Graduação em Saúde Pública do Departamento de Saúde Pública da UFSC.

${ }^{3}$ Odontólogo. Doutor em Enfermagem Psiquiátrica. Professor Adjunto do Programa de Pós-Graduação em Saúde Pública do Departamento de Saúde Pública da UFSC.

Autor correspondente:

Rodrigo Otávio Moretti-Pires

Universidade Federal de Santa Catarina

Rua Roberto Sampaio Gonzaga, s/n - 88040-970 - Florianópolis-SC, Brasil

Email: rodrigomoretti@ccs.ufsc.br

Recebido: 09/11/09

Aprovado: 19/03/10 


\section{INTRODUÇÃO}

No final da década de 1970, foi organizado o Movimento da Reforma Sanitária, que envolveu diversos setores da sociedade e desencadeou um novo modelo de atenção à saúde. Decorrente da grande participação deste movimento, aconteceu em 1986 a $8^{\text {a }}$ Conferência Nacional de Saúde que consolidou a ideia do Sistema Único de Saúde (SUS) e fundamentou os artigos sobre saúde da Constituição Nacional de 1988, regulamentados apenas em 1990, pelas Leis Orgânicas da Saúde(LOS) n. 8.080/90 e n. 8.8142/90. Fruto político da articulação popular e de pensadores da época, as mudanças oficializadas basearam-se nas necessidades relacionadas às práticas de saúde da época ${ }^{(1)}$.

Este novo sistema tem por finalidade formular e executar políticas econômicas e sociais no campo da saúde, na redução do risco de doenças e outros agravos, e assegurar condições que resultem em um acesso universal e igualitário às ações e serviços. Paralelamente, o sistema de saúde deve visar a prevenção, a proteção e a restituição da saúde de todos os cidadãos brasileiros, além de incluir em seus princípios o controle social, que proporciona à sociedade a participação na gestão, por meio dos representantes inseridos nos Conselhos Locais de Saúde, com vistas a formular estratégias quanto ao controle da operacionalização da política de saúde ${ }^{(1-2)}$.

Como estratégia na superação de barreiras à consolidação dos preceitos e diretrizes do SUS, foi criado em 1994 o Programa de Saúde da Família (PSF) - somando-se ao Programa de Agentes Comunitários da Saúde (PACS), criado em 1991 - com a intenção de reorganizar o modelo assistencial brasileiro. Em 2006, o PSF foi adotado como modelo assistencial brasileiro, a partir de então denominado Estratégia de Saúde da Família (ESF) ${ }^{(3)}$.

Cabe ressaltar que aproximar a prestação de serviços de atenção à saúde a todos os cidadãos é um dos preceitos fundamentais do Movimento de Reforma Sanitária Brasileira. O novo modelo assistencial se construiu sobre diferentes bases e critérios de processo de trabalho, substituindo o trabalho voltado somente à assistência curativa e hospitalar, mas ampliando o foco para a prevenção de doenças e promoção da saúde ${ }^{(3)}$. A ESF apresenta a peculiaridade de ser constituída por equipes multiprofissionais, compostas por um médico, um enfermeiro, um cirurgião-dentista, um auxiliar de consultório dentário, um ou dois auxiliares de enfermagem e de quatro a seis agentes comunitários de saúde (ACS) $)^{(4)}$.
O ACS, profissional até então não existente, é selecionado de acordo com as áreas de responsabilidade da Equipe de Saúde da Família. Ele deve morar na comunidade e ter uma relação de proximidade com as famílias assistidas, para que possa estabelecer vínculo e imergir nas condições de saúde da comunidade adscrita e subsidiar o trabalho dos demais membros da equipe ${ }^{(4)}$. Entre seus atributos, o ACS deve exercer capacidade de liderança para direcionar suas ações à melhoria das condições de saúde e de vida da população, além de ser responsável por uma média de cento e cinquenta famílias que residem em seu território de abrangência, se na área urbana, e duzentas famílias na área rural, próximo da realidade de cada uma. Muitas das ações se dão através da visita domiciliar, fornecendo orientações e encaminhando os usuários para a Unidade Básica de Saúde (UBS) em casos necessários, ou propiciando, de outras formas, uma melhora na qualidade de vida dos mesmos ${ }^{(5)}$.

Neste processo de reorientação do modelo assistencial a que se propõe o SUS, a realidade geográfica das populações do interior do Estado do Amazonas constitui-se barreira à democratização da possibilidade de utilização dos serviços de saúde, que se concentram, na maioria das vezes, nas áreas urbanas.

Na realidade amazônica, não apenas o acesso, mas diversos aspectos do SUS se configuram de maneira peculiar em relação a outras partes do Brasil. Os serviços de saúde prestados àquela população são precários e isso resulta de questões maiores relacionadas à condição social e histórica produzida pela exclusão, emblematizada na impossibilidade da plena realização dos indivíduos e de acesso a recursos que lhes melhorariam a vida ${ }^{(6)}$.

Diante do exposto, o presente artigo objetiva apreender a percepção dos profissionais sobre o funcionamento da ESF no interior da Amazônia.

\section{PERCURSO METODOLÓGICO}

Trata-se de uma pesquisa qualitativa, de caráter exploratório, que se baseou na busca da compreensão sobre o contexto de vida, numa perspectiva de experiência pessoal de cada um dos sujeitos envolvidos, valorizando as características e dinâmicas de determinado fenômeno sem a prerrogativa da

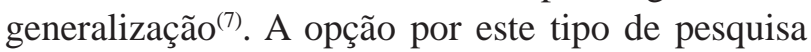
justifica-se por sua capacidade de incorporar significado e intenção aos atos, às relações e às estruturas sociais.

Cogitare Enferm. 2010 Abr/Jun; 15(2):231-7 
Essas últimas são tomadas tanto no seu advento como na sua transformação, como construções humanas significativas $^{(8-9)}$, na tentativa de compreender um problema na perspectiva dos sujeitos que o vivenciam ${ }^{(7)}$.

O estudo foi realizado no período de janeiro a março de 2009, no município de Coari, Região do Médio Solimões, situado geograficamente no centro do Estado do Amazonas, à distância de 363 quilômetros da cidade de Manaus. Neste município existiam 11 unidades de SF, uma para cada equipe, com cobertura de $100 \%$ da população urbana. O município contava com 11 equipes de Saúde da Família, duas unidades fluviais de atenção, um hospital de adscrição regional e um campus avançado da Fundação de Medicina Tropical. A equipe de saúde do SUS era composta por 28 médicos, 35 enfermeiros, 14 odontólogos, dois nutricionistas, dois psicólogos, três assistentes sociais, três fisioterapeutas e 223 ACS, dos quais 120 (53,8\%) atuavam na zona urbana.

Foram utilizadas como critérios de inclusão dos sujeitos as seguintes características: (1) profissionais que trabalhassem na atenção primária em UBS com ESF; (2) a UBS analisada devia estar em funcionamento há pelo menos quatro anos. Participaram do estudo dois odontólogos, um nutricionista, dois enfermeiros, seis ACS, um auxiliar de enfermagem, dois médicos, três recepcionistas, um técnico em enfermagem, um servente e um agente administrativo da farmácia.

Todos os entrevistados participaram, após explicação, compreensão e registro formal de consentimento livre e esclarecido. Assim, o presente estudo respeitou as normas de pesquisa envolvendo seres humanos com base na Resolução n. 196/96 do Conselho Nacional de Saúde, sendo aprovado pelo Comitê de Ética em Pesquisa da Universidade Federal do Amazonas sob o protocolo 054/07 (CAAE 0055.0.115.000-07).

A coleta de dados foi realizada por meio de entrevista semiestruturada, com questões que se referiam ao funcionamento da UBS e às ações desenvolvidas pela equipe e realizadas na própria unidade. As informações foram registradas em gravador digital e, em seguida, transcritas na íntegra, seguindo os passos metodológicos: a leitura exaustiva e a categorização dos dados, esta obtida através das reincidências de informações contidas nos depoimentos dos diversos sujeito $^{(8)}$.

Em seguida, a análise buscou categorias de discursos com mesmo tema, a partir da perspectiva dos profissionais de saúde inseridos na ESF sobre o funcionamento e o cotidiano. Após esta fase interpretativa, realizou-se a busca de contradições e coerências, de forma que o processo de análise fundamentou-se por um pensamento hermenêutico dialético, compreendendo de maneira crítica os achados ${ }^{(7)}$. Como fundamentação teórica foram utilizados os documentos oficiais que definem os pressupostos do trabalho em Saúde da Família, dentro da Política Nacional de Atenção Básica $^{(3)}$.

\section{RESULTADOS}

Um importante achado que emergiu do domínio empírico refere-se ao fato da assistência à saúde restringir-se aos aspectos curativos e imediatos das necessidades de saúde da população adscrita na ESF deste contexto amazônico. Chama a atenção que estes aspectos, além de puramente biológicos, também se referem à livre demanda por atendimento na UBS, que se expressa na seguinte fala:

[...] o atendimento é muito rápido e eficaz e, dentro das necessidades, faz-se os encaminhamentos, dando prioridade realmente para quem precisa. (Enfermeiro X)

Outra categoria referiu-se a longitudinalidade no acompanhamento da saúde dos usuários pela equipe. A dificuldade para fixação da mão-de-obra na região é um fator implicado neste contexto, que explica a alta rotatividade dos profissionais dentro da rede assistencial no município, havendo falhas no cumprimento desta diretriz da ESF. Este panorama apresenta prejuízos de caráter intersubjetivos entre profissional e usuário, dificultando o estabelecimento de vínculo, como se percebe a seguir:

[...] muitas vezes o médico, o enfermeiro ou o dentista não ficam em uma só UBS ao longo do tempo. Sempre estão trocando de UBS, ou vão embora para outra cidade. (Auxiliar de Enfermagem)

[...] o que deixa o paciente um pouco inseguro é por que há muita troca de profissional no Posto [...]. (Recepcionista Y)

As falas analisadas relatam que o ACS possui papel fundamental na atenção primária à saúde. Eles são os profissionais que reconhecem os problemas de saúde dos indivíduos e da comunidade que assistem, que realizam diagnósticos de saúde da comunidade 
repassando-os para os demais profissionais da UBS, e conhecem os membros de cada família gerando maior aproximação entre serviços de saúde/familiares. Ao mesmo tempo, a proximidade dos demais profissionais junto à comunidade é frágil, como se apenas o ACS fosse responsável por isso ou que os profissionais fossem responsáveis apenas pela doença do usuário:

[...] eles que fazem a ponte entre os profissionais $e$ as famílias, pois eles já estão mais próximos delas [...]. (Nutricionista)

[...] como os ACS andam pela comunidade e muitos procuram a UBS, então dá para se saber dos problemas que estão ocorrendo na comunidade. (Assistente Administrativo Y)

[...] no posto o médico não conhece os membros da minha família, pois são muitos, apenas os ACS, porque estão fazendo sempre as visitas em minha casa. (Usuário X)

Por fim, a burocratização da Secretaria Municipal de Saúde, sem participação ativa nos processos locais foi denunciada pelos entrevistados. Há pálida uma participação da gestão municipal junto às equipes, tanto no suporte às demandas locais como no monitoramento das ações, em uma perspectiva ultrapassada de Gestão:

[...] muitos deles só querem mesmo saber do seu salário, não estão nem aí pra necessidade das famílias. (Auxiliar de Enfermagem)

[...] a pessoa responsável nunca comparece na Unidade de Saúde. Quando aparece é pra pedir papelada. (Agente comunitário de saúde Z).

O quadro 1 relaciona os achados empíricos com

Quadro 1- Análise dos pressupostos da ESF com as categorias dos conteúdos empíricos e as contradições entre estes. Coari, 2009.

\begin{tabular}{|c|c|c|c|}
\hline Categoria empírica & $\begin{array}{l}\text { Pressupostos da } \\
\text { Saúde da Família }\end{array}$ & $\begin{array}{l}\text { Conteúdo empírico } \\
\text { das falas }\end{array}$ & Contradições \\
\hline $\begin{array}{c}\text { Assistência curativa e } \\
\text { imediata das necessidades } \\
\text { da livre demanda que } \\
\text { procura a UBS }\end{array}$ & $\begin{array}{l}\text { Saúde da Familia } \\
\text { pressupõe atendimento } \\
\text { amplo, na perspectiva } \\
\text { ampliada em saúde }\end{array}$ & $\begin{array}{c}\text { Atendimento rápido à } \\
\text { necessidade imediata, } \\
\text { por livre demanda, distante } \\
\text { de um atendimento } \\
\text { contínuo e a longo prazo }\end{array}$ & $\begin{array}{l}\text { Cuida-se da cura, mas não da } \\
\text { prevenção específica e da } \\
\text { promoção da saúde }\end{array}$ \\
\hline $\begin{array}{c}\text { Falta de longitudinalidade } \\
\text { no acompanhamento } \\
\text { aos usuários }\end{array}$ & $\begin{array}{l}\text { O profissional deve criar } \\
\text { vínculo com } \\
\text { a comunidade }\end{array}$ & $\begin{array}{l}\text { Os profissionais não ficam no } \\
\text { município ou, se ficam, rodam } \\
\text { de unidade em unidade para } \\
\text { suprir a falta de profissionais }\end{array}$ & $\begin{array}{l}\text { Desconhecimento do contexto } \\
\text { da comunidade que se atende }\end{array}$ \\
\hline $\begin{array}{c}\text { O ACS é o profissional } \\
\text { que efetivamente imerge na } \\
\text { vida comunitária }\end{array}$ & $\begin{array}{l}\text { Os profissionais devem } \\
\text { realizar periodicamente } \\
\text { visitas domiciliares e } \\
\text { ações junto à } \\
\text { comunidade }\end{array}$ & $\begin{array}{c}\text { Apenas o ACS vai até a } \\
\text { comunidade com regularidade }\end{array}$ & $\begin{array}{l}\text { Falta de ação integrada entre } \\
\text { os profissionais como equipe } \\
\text { e destes com a comunidade }\end{array}$ \\
\hline $\begin{array}{l}\text { Papel simplesmente } \\
\text { burocrático do } \\
\text { Gestor Municipal }\end{array}$ & $\begin{array}{l}\text { Suporte para as } \\
\text { demandas pela } \\
\text { Gestão Municipal }\end{array}$ & $\begin{array}{l}\text { Gestão focada apenas nas } \\
\text { questões burocráticas }\end{array}$ & $\begin{array}{l}\text { A falta de suporte da Gestão } \\
\text { para o nível local legitima } \\
\text { o ciclo de ausências }\end{array}$ \\
\hline
\end{tabular}

os pressupostos da Política Nacional de Atenção Básica ${ }^{(3)}$, pertinentes às categorias que emergiram na perspectiva dos entrevistados.

\section{DISCUSSÃO}

O sistema de saúde vigente preconiza a interdisci- plinaridade. Para a sua existência, há necessidade de profissionais que interajam e se comuniquem por meio de seus diferentes saberes. Porém, é percebido que na Atenção Básica, a equipe tem dificuldade de se relacionar devido aos níveis hierárquicos, havendo certa fragmentação do trabalho, o que ocasiona contradições. Com os profissionais desempenhando sua função sem 
ter clareza das prioridades e objetivos da instituição ou município, as ações e o bom entrosamento da equipe são prejudicados ${ }^{(10-11)}$.

Estes achados implicam na discussão da categoria “cuidado", re-significada na literatura atual em direção às dimensões sociais e políticas, assim como no aspecto da mediação entre ajuda e poder de ajudar, implicando na autonomia dos usuários ${ }^{(12-13)}$. Os dados do estudo apresentam a dificuldade dos profissionais em ouvir demandas dos pacientes e, por consequência, tratá-los como sujeitos, sendo o "cuidado" centrado nos protocolos de saúde e não no usuário ${ }^{(12)}$. Na ESF, a produção de serviços de saúde não deveria se distanciar da noção do usuário como sujeito histórico, de conhecimentos próprios e tão importantes quanto os saberes dos profissionais de saúde.

O planejamento das ações interdisciplinares deveria ser feito em função das necessidades do grupo populacional a ser atendido, não se limitando apenas ao papel de cada profissional. No entanto, as informações encontradas apontam para um processo diferente deste, cabendo apenas ao ACS a real imersão na complexidade da comunidade, enquanto o trabalho dos demais profissionais se mostra desarticulado e focado apenas na cura. A satisfação junto aos serviços irá resultar em maior adesão dos pacientes ao tratamento, através do vínculo e da interrelação profissionalpaciente-família e das respostas às necessidades de saúde do usuário ${ }^{(11-14)}$.

Defende-se na literatura que, para haver a relação multiprofissional, são necessárias atitudes entre os profissionais, como o respeito e o reconhecimento da importância de cada profissão, tolerância, aceitação de sugestões, comprometimento com o sistema público, atitude de mudança, humildade, respeito às diferenças, ética, liderança e empatia. Com a prática destas atitudes, existiria maior interdisciplinaridade entre as profissões, gerando tratamentos mais eficazes ${ }^{(10)}$.

A ESF presume a utilização da assistência domiciliar à saúde, como forma de viabilizar a inserção e o conhecimento do profissional sobre a realidade de vida da população, bem como a criação de vínculos com a mesma $^{(15)}$.

Existem indícios de que a chegada de um profissional da ESF ao domicílio promove o bem estar da população, mesmo sem uma intervenção mais específica da equipe ${ }^{(16)}$. Por outro lado, existe uma preocupação com a postura profissional, pois é necessário respeito à privacidade da pessoa e sua cultura, bem como o exercício da ética, fazendo com que ocorra uma prática intervencionista coerente e que dê ênfase à liberdade da pessoa em fornecer somente as informações que desejar ${ }^{(15)}$.

De acordo com o Ministério da Saúde, as funções de uma equipe de saúde consistem em: conhecer as famílias e prestar assistência integral pelas quais estão responsáveis, com prioridade nas suas características econômicas, culturais, sociais, epidemiológicas e demográficas; identificar as enfermidades e situações de risco mais recorrentes em sua população; elaborar, com a participação da comunidade, um plano local para o combate aos problemas de saúde e fatores que colocam em risco a mesma; garantir acesso e continuidade de tratamento; promover ações intersetoriais; estimular a participação popular, discutindo com a comunidade questões relacionadas aos conceitos de cidadania, de direito à saúde e suas bases legais, além de realizar visitas domiciliares. Dentre os membros integrantes da ESF, o ACS atua como mediador entre a equipe e as necessidades de sua comunidade, cuja seleção deve ser feita segundo a Lei 10.507, de 10 de julho de 2002, que criou esta mesma profissão ${ }^{(1)}$.

Dada a complexidade da prestação de serviços de saúde, é fundamental que a gestão se comprometa com o dia-a-dia das equipes, em razão das redes complexas existentes nos serviços de saúde ${ }^{(16)}$. Mais do que gerenciar recursos e dados burocráticos dos sistemas de informação, a Gestão Municipal deveria partir do conhecimento dos problemas de saúde de cada Equipe de Saúde da Família, para a condução da gestão em si, objetivando a mudança efetiva nas práticas em saúde no âmbito da Atenção Primária, uma vez que até mesmo a educação em saúde praticada refere-se ao paradigma tradicional e bancário ${ }^{(17)}$.

Muitas das dificuldades assumidas pelos entrevistados referem-se ao componente individual da postura do profissional, mas em muito também as dificuldades estruturais da organização do próprio SUS, panorama reforçado pela participação do Gestor Municipal apenas como executor de burocracias administrativas da Gestão em Saúde.

\section{CONSIDERAÇÕES FINAIS}

A despeito das dificuldades encontradas pelos profissionais sobre o funcionamento da ESF no interior da Amazônia, não foi encontrada falta de crença na Saúde da Família como instrumento de melhoria da saúde da população.

A prática da ESF no interior da Amazônia - e 
talvez no restante do Brasil - ainda fundamenta-se na assistência curativa e imediata das necessidades da livre demanda que procura a UBS, apesar das potencialidades para novas modalidades de intervenção comunitárias, dada a posição privilegiada das equipes na identificação das necessidades de saúde da população adscrita.

Ainda se preserva a atuação tradicional dos profissionais, com certa centralidade do ACS no conhecimento da comunidade. O envolvimento do ACS nas ações de saúde tem o intuito de estabelecer uma postura participativa, informativa e de vínculo com as famílias da sua área de abrangência. Este profissional apresenta importância fundamental como agente transformador no processo saúde-doença. Por outro lado, os demais profissionais não têm inserção significativa junto às dinâmicas da comunidade, o que fere os pressupostos da ESF.

Por fim, o sistema de saúde ainda necessita de reformulação em termos de processo de trabalho, principalmente no que cabe aos aspectos de gestão e monitoramento das ações na atenção primária, de forma a torná-la mais efetiva, com co-responsabilização entre usuários, equipe e gestores, o que não foi encontrado no campo investigado.

Há que se refletir em quanto estes achados diferem de outros contextos no Estado e mesmo no Brasil, haja vista que a literatura traz padrões similares em outras partes do país, sugerindo-se investigações que avaliem peculiaridades regionais e as confrontem com os dados que emergirem do processo de trabalho em Saúde da Família.

\section{REFERÊNCIAS}

1. Fleury S. Reforma sanitária brasileira: entre o instituinte e o instituído. Ciênc Saúde Colet. 2009;14(3):743-52.

2. Ceccim RB. Educação e saúde: ensino e cidadania como travessia de fronteiras. Trab Educ Saúde 2009;6(3):44356.

3. Brasil. Portaria n. 648, de 28 de março de 2006. Dispõe sobre a revisão de diretrizes e normas para a organização da Atenção Básica para o Programa Saúde da Família (PSF) e o Programa de Agentes Comunitários de Saúde (PACS). Diário Oficial da República Federativa do Brasil, Brasília, 2006. Seção X:X.

4. Seoane AF, Fortes PAC. A percepção do usuário do Programa Saúde da Família sobre a privacidade e a confidencialidade de suas informações. Saúde Soc. 2009;18(1):42-9.

5. Carvalho YM, Ceccim RB. Formação e educação em saúde: aprendendo com a saúde coletiva. In: Campos, G; Minayo MCS, Arkeman M, Drumond Júnior M, Carvalho YM, organizadores. Tratado de saúde coletiva. São Paulo - Rio de Janeiro: HUCITEC/Fiocruz; 2006. p.151-60.

6. Ranciaro MMMA. Andirá: memórias do cotidiano e representações sociais. Manaus: EDUA; 2004.

7. Leopardi MT. Metodologia da pesquisa na saúde. $2^{\mathrm{a}}$ ed. Florianópolis: UFSC/Pós-graduação em Enfermagem; 2002.

8. Crotty M. The foundations of social research: meaning the perspective in the research process. London: Sage Publications; 2003.

9. Minayo MCS. O desafio do conhecimento: pesquisa qualitativa em saúde. São Paulo-Rio de Janeiro: HUBITEC-ABRASCO; 1999.

10. Bagnato MHS, Renovato RD, Bissenell. De interdisciplinaridade e multireferencialidade na educação superior em saúde. Cogitare Enferm. 2007;12(3):365-70.

11. Garcia MAA, Pinto ATBC, Odoni APC, Longhi BS, Machado LL, Linek MS, et al. A interdisciplinaridade necessária à educação médica. Rev Bras Educ Med. 2007;31(2):147-55.

12. Pires MRGM. Politicidade do cuidado e avaliação em saúde: instrumentalizando o resgate da autonomia de sujeitos no âmbito de programas e políticas de saúde. Rev Bras Saúde Mater Infant. 2005;5 Supl 1:S71-S81.

13. Silva AG, Merhy EE, Carvalho LC. Refletindo sobre o ato de cuidar da saúde. In: Pinheiro R, Matto RA, organizadoras. Construção da integralidade: cotidiano, saberes e práticas em saúde. Rio de Janeiro: UERJ, IMS, ABRASCO; 2007.p.115-130.

14. Oliveira MC. Os modelos de cuidados com o eixo de estruturação de atividades interdisciplinares e multiprofissionais em saúde. Rev Bras Educ Med. 2008; 32(3):347-55.

15. Giacomozzi CM, Lacerda MR. A prática da assistência domiciliar dos profissionais da estratégia de saúde da família. Texto Contexto Enferm. 2006;15(4):645-53. 
16. Pitta AMR, Rivera FJU. Sobre pontos de partida: planejamento em comunicação e integralidade da atenção em saúde. Interface - Comunic Saúde Educ. 2006;10(2): 395-410.

17. Maciel MED, Borges PKO, Sales EM, Renovato RD. Educação em saúde na percepção de Agentes Comunitários de Saúde. Cogitare Enferm. 2009; 14(2):340-5. 